\title{
Anti-inflammatory fibrosis suppression in threatened trabeculectomy bleb failure produces good long term control of intraocular pressure without risk of sight threatening complications
}

\author{
J R Fuller, T H Bevin, A C B Molteno, B J T Vote, P Herbison
}

Br J Ophthalmol 2002;86:1352-1355

\begin{abstract}
Aims: To determine the long term outcome of systemic anti-inflammatory fibrosis suppression in cases of threatened trabeculectomy bleb failure in open angle glaucoma. Methods: This prospective non-comparative case series followed 77 eyes of 63 patients which showed signs of threatened early bleb failure and were treated with oral anti-inflammatory fibrosis suppression of prednisone, a non-steroidal anti-inflammatory agent, and colchicine taken for a mean period of 6 weeks, in addition to standard postoperative topical treatment, for a mean follow up of 6 years.

Results: Trabeculectomy with anti-inflammatory fibrosis suppression controlled the IOP at $\leqslant 21 \mathrm{~mm} \mathrm{Hg}$ with a probability of $0.91(95 \% \mathrm{Cl}: 0.81$ to 1.0$)$ at 8 years and 0.89 (95\% Cl: 0.56 to 1.1$)$ at 12 years. There were no reported cases of endophthalmitis, hypotonous maculopathy, late bleb leak, or serious systemic side effects.

Conclusion: Anti-inflammatory fibrosis suppression provided good control of bleb fibrosis without risk of sight threatening complications in a patient group at high risk of bleb failure.
\end{abstract}

$\mathrm{D}$ espite the variety in technique, all drainage blebs undergo distinct stages in formation that correlate with normal wound healing events for these tissues ${ }^{1-3}$ and involve a characteristic sequence of changes in the episcleral tissues and conjunctiva in response to the presence of aqueous. ${ }^{4}$ The early trial of Molteno implants involved cases of terminal glaucoma in young adults in which implant insertion produced a period of intense bleb inflammation with transient elevation of intraocular pressure (IOP) to between $30-50 \mathrm{~mm}$ Hg. ${ }^{5}$ It was established that IOP elevation reflected the tissue pressure in the inflamed bleb wall and that administration of systemic steroids for 6 weeks after implant insertion would shorten the period of bleb inflammation and its associated elevation of IOP. This produced a less fibrosed bleb and gave better long term control of IOP. Further clinical trials showed that steroids, even in massive doses, could not be relied on to give adequate control of bleb inflammation and fibrosis in all cases. $^{46}$ The limited effect of systemic steroids led one of us (ACBM) to introduce a synergistic combination of oral prednisone, fluphenamic acid, and colchicine to prevent bleb inflammation and fibrosis in selected cases after drainage by trabeculectomy or Molteno implant. ${ }^{4}$

This communication reports the experience with the use of anti-inflammatory fibrosis suppression regimen of prednisone, non-steroidal anti-inflammatory agents, and colchicine in the management of threatened bleb failure after trab- eculectomy at Dunedin Hospital in 77 eyes of 63 patients operated on between 1978 and 1998.

\section{PATIENTS AND METHODS \\ The setting}

The ophthalmology department at Dunedin Hospital is the centre for all public ophthalmic services for the province of Otago. This concentration of services in one department and the stable population of this region have facilitated long term follow up of patients.

\section{Data collection}

All patients with primary open angle glaucoma who underwent trabeculectomy were identified from the computerised database of the Otago Glaucoma Surgery Outcome Study. Patients who received anti-inflammatory fibrosis suppression for threatened bleb failure and had not been treated with fluorouracil or mitomycin $\mathrm{C}$ were selected and their notes retrieved. Data were collected from both the computer database and the clinical notes. Consent for data retrieval was obtained from the ethics committee of the regional health authority.

\section{Data recorded preoperatively}

The results of the preoperative history and clinical examination were recorded in a standardised format. This included a detailed inventory of the ocular findings on slit lamp examination and ophthalmoscopy and measurements of the visual acuity and IOP.

Visual acuity was measured on a standard Snellen type logMAR chart incorporating 12 lines from 20/15 to 20/200. The IOP was measured using a Haag-Streit Goldmann applanation tonometer (Haag-Streit AG, Koniz, Switzerland) mounted on a slit lamp. Local and systemic ocular medications prescribed, including hypotensive and anti-inflammatory agents were recorded. Details of the patient's major general medical conditions were also recorded with particular emphasis on diabetes, vascular disease, hypertension, renal function, respiratory disease, alcoholism, malignant disease, and major illnesses.

\section{Operative data recorded}

Operative technique and any intraoperative complications were recorded.

\section{Postoperative follow up}

Patients were examined by slit lamp and direct ophthalmoscopy at each visit. All cases were routinely seen the day after surgery. Routine follow up visits were scheduled 1, 3, 7, and 9 weeks after surgery however the cases in this series with signs of early bleb failure were followed more frequently at 1-2 week intervals during the anti-inflammatory fibrosis suppression therapy. Thereafter, visits were scheduled every 3 months 
for the first year, every 6 months for the next 2 years, and then at least annually. Any postoperative complications and further ocular surgery were recorded. Patients who moved out of the district were referred to local ophthalmologists for follow up. Patients who "disappeared" from follow up were traced through their general medical practitioners, the voters' roll, and cemetery records in order to determine their current whereabouts or date of death.

\section{Definition of end points}

The preoperative IOP for each eye was taken as the mean IOP in the month before surgery. Postoperatively IOP was taken as the mean for each postoperative year. When more than a year elapsed between postoperative visits, intervening values were interpolated. The medications used were recorded at each postoperative visit.

In this paper "control" was defined as a persistent IOP of $\leqslant 21 \mathrm{~mm} \mathrm{Hg}$ with or without hypotensive medication. "Failure" was defined as a persistent IOP of $>21 \mathrm{~mm} \mathrm{Hg}$, further glaucoma surgery or enucleation. The time of failure was taken as the first date at which any of these events was recorded.

The preoperative visual acuity for each patient was taken as the best corrected visual acuity in the month before surgery. The visual acuity at final follow up was taken as the best visual acuity during the final year of follow up.

\section{Surgical technique}

A standard Cairns' type trabeculectomy with a guarded filter and limbal based conjunctival flap was used in 68 eyes while a fornix based flap was used in the remaining nine eyes. Topical postoperative antibiotics, steroids, and mydriatics were administered for 4-6 weeks.

\section{Anti-inflammatory fibrosis suppression}

Anti-inflammatory fibrosis suppression was commenced when the presence of increased vascularity, contraction of the suture line and/or Tenon's cyst in combination with a rising IOP suggested impending bleb failure. Specific signs used to evaluate the need for anti-inflammatory fibrosis suppression included an absence of subconjunctival drainage in the early postoperative period with high IOP, marked vasodilation of bleb blood vessels that was not fully reversed by topical adrenaline (epinephrine) eye drops $1 \%$ and accompanied by higher than expected IOP, undue reduction in the area of the bleb with higher than expected IOP, and visible deposition of fibrous tissue or the formation of an overt Tenon's cyst. The dosages of prednisone, non-steroidal anti-inflammatory agent, and colchicine were individually tailored to patient size, age, and general state with higher doses being given to young, less than 65-70 years, patients or those showing more marked signs of bleb failure. Since earlier studies had shown that intermittent (once daily) administration of prednisone completely prevented the antifibrotic action of the antiinflammatory fibrosis suppression regimen, care was taken that all medications were prescribed and taken at regular intervals three times a day in order to maintain adequate blood levels throughout the 24 hour period.

\section{RESULTS}

The 77 eyes in this series were drawn from the 551 trabeculectomies with primary open angle glaucoma performed at Dunedin Hospital between 1978 and 1998. There was no significant difference with respect to age at operation, preoperative IOP, number of hypotensive medications, preoperative visual acuity, extent of field loss, optic disc cupping, or surgical technique (limbus based or fornix based flaps) between cases that received anti-inflammatory fibrosis suppression and those that did not.
The mean age at operation of the anti-inflammatory fibrosis suppression series was 66 years (range 32-82 years). There were 48 male eyes and 29 female eyes, and 43 right and 34 left eyes. Of the 63 different patients 14 had trabeculectomies in both right and left eyes. There were 57 cases of chronic simple open angle glaucoma and 20 cases of pseudoexfoliative glaucoma. The mean length of follow up was 6.0 years (range $0.5-$ 21.5 years). Ten eyes ( $13 \%$ ) were lost to follow up after having been followed for a mean of 9.2 years (range 2.9-17.5 years).

The mean preoperative IOP was $25 \mathrm{~mm} \mathrm{Hg}$ (range 15-47 $\mathrm{mm} \mathrm{Hg}$ ). Two patients had previously undergone trabeculectomy, six cataract surgery, and three had had laser trabeculoplasty. The mean number of preoperative glaucoma medications was 1.9 (range 0-4).

Four patients began anti-inflammatory fibrosis suppression within a week before surgery while the remainder commenced it at an average of 11 days after surgery (range -7-30 days postoperatively). One further case commenced antiinflammatory fibrosis suppression 80 days postoperatively in conjunction with bleb needling. The mean IOP before commencement of anti-inflammatory fibrosis suppression was $20 \mathrm{~mm} \mathrm{Hg}$ (range $5-48 \mathrm{~mm} \mathrm{Hg}$ ).

\section{Anti-inflammatory fibrosis suppression}

Cases were prescribed prednisone $2.5 \mathrm{mg}, 5 \mathrm{mg}$, or $10 \mathrm{mg}$ three times a day (mean daily dose $19 \mathrm{mg}$ ). Colchicine, $0.2 \mathrm{mg}$ or 0.3 mg three times a day, was prescribed (mean daily dose 0.9 $\mathrm{mg})$. The non-steroidal anti-inflammatory agent used was fluphenamic acid 100-200 mg three times a day in the first 29 cases. When fluphenamic acid became unavailable diclofenac sustained release $100 \mathrm{mg}$ daily or $50 \mathrm{mg}$ three times a day was prescribed in the next 46 cases (mean daily dose $100 \mathrm{mg}$ ) and tenoxicam $20 \mathrm{mg}$ daily was prescribed in four cases. Treatment was prescribed for a mean of 6 weeks (range 1-11 weeks).

\section{Response to anti-inflammatory fibrosis suppression}

The response to anti-inflammatory fibrosis suppression was consistent with no obvious changes to the drainage bleb noted in the first 2-3 days after beginning treatment. On day 3-4 there was a marked reduction in the vascularity of the bleb often accompanied by extension of the bleb (aqueous spread) and typically by a marked reduction in IOP. Four patients experienced marked reduction in IOP with shallowing of the anterior chamber at this time. The medication was temporarily discontinued resulting in restoration of the anterior chamber after 1-3 days. The response in IOP was greater the earlier the regimen was started after surgery (within 1-2 weeks). When started later (3-4 weeks) the response was less marked but none the less there was a noticeable reduction in bleb vascularity. Six cases went on to develop fibrosed blebs by 4-11 weeks, and were needled while on anti-inflammatory fibrosis suppression. Needling resulted in extension of the blebs with control of IOP in all cases.

\section{Side effects of anti-inflammatory fibrosis suppression}

There were no cases of endophthalmitis, hypotonous maculopathy, late bleb leak, or serious systemic side effects. Nine patients had minor systemic side effects. Seven patients were placed on oral histamine-2 blockers (ranitidine), one of which had a 1 week break in treatment. One patient had 4 weeks of topical diclofenac owing to problems with oral diclofenac during an 8 week treatment course.

\section{Outcome}

Intraocular pressure control was achieved in 69 eyes of which 19 were on hypotensive medication at final follow up. There were eight failures. The annual mean IOP was $16.36 \mathrm{~mm} \mathrm{Hg}$ at 1 year postoperatively and remained between 15.3-13.9 mm Hg for 15 years of follow up. The Kaplan-Meier probability of 


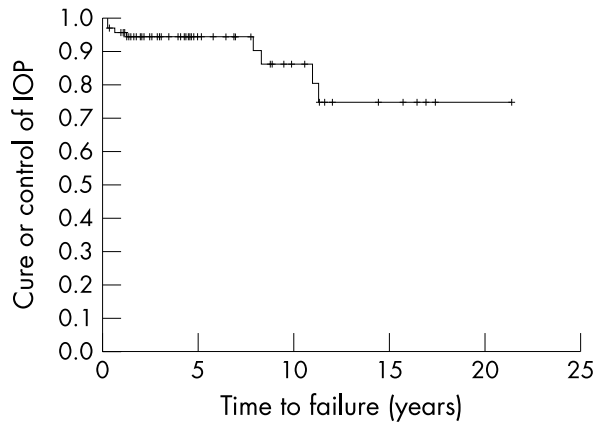

Figure 1 Kaplan-Meier life table demonstrating probability of control of intraocular pressure with time.

IOP control was 0.91 ( $95 \%$ CI: 0.81 to 1.0 ) at 8 years and 0.89 (95\% CI: 0.56 to 1.0 ) at 12 years (see Fig 1). Preoperatively, 61 patients had a visual acuity of 6/9 or better and 16 were worse than 6/9. At final follow up 51 had a visual acuity of $6 / 9$ or better and 26 were worse than 6/9. Eight eyes (10.4\%) later underwent cataract extraction. Progressive field loss occurred in 19 eyes $(24.7 \%)$ compared to 119 of the $463(25.7 \%)$ matched eyes in which anti-inflammatory fibrosis suppression was not used.

\section{Intraocular pressure control group}

Of the 19 who achieved IOP control with hypotensive medication at final follow up, 17 were on one treatment and two on two treatments (mean 0.3 for IOP control group). Fourteen eyes underwent further surgery. One eye had early anterior chamber reformation, eight eyes underwent cataract extraction and lens implant, and six eyes had trabeculectomy manipulations or bleb needling. One patient after 10 years of good IOP control developed a retinal detachment after cataract surgery. This subsequently redetached and the eye became blind.

\section{Failure group}

Six of the eight failures subsequently had Molteno implants inserted. Two of these implants were inserted early (within 2 months) in the right and left eyes of a patient who did not form a drainage bleb at any time postoperatively. The remaining four implants were inserted in cases of gradual failure at $2,4,5$, and 10 years postoperatively. All cases which later underwent Molteno implant insertion had IOP control of $<21$ $\mathrm{mm} \mathrm{Hg}$ on no treatment 3 months after trabeculectomy. One patient developed a central retinal vein occlusion and thrombotic glaucoma and the eye was eventually enucleated. Another patient developed a retinal detachment 12 years after trabeculectomy and despite three vitreoretinal procedures the eye was eventually enucleated a year after the first detachment procedure. There was no significant difference in the failure rate of eyes with and without pseudocapsular exfoliation or when one eye of bilateral cases was excluded from analysis.

\section{DISCUSSION}

This case series documents the outcome of anti-inflammatory fibrosis suppression in the management of the failing trabeculectomy bleb. The main cause of glaucoma surgery failure is scarring at the episcleral wound. Although scarring is the normal response to a physical insult the intensity of the clinical healing response is individually specific and relatively low risk cases may still fail. ${ }^{1-3}$

The application of antimetabolites in the perioperative or postoperative period to minimise the healing response has become common since the introduction of 5-fluorouracil in 1983. 5-Fluorouracil has been shown to improve glaucoma outcome in high risk patients when given postoperatively. ${ }^{89}$ Mitomycin C has a more potent effect than 5-fluorouracil and has been shown to improve glaucoma outcome in high risk cases when used postoperatively. ${ }^{10}$ However, because of their long term local impairment of ocular healing and defence both these antimetabolites, and particularly mitomycin $\mathrm{C}$, have serious sight threatening side effects. Late bleb leak has been reported in $8 \%$ of cases and hypotonous maculopathy in $4 \%$ of cases with the perioperative use of 5-fluorouracil. ${ }^{11} \mathrm{~A}$ study of the follow up and complications of perioperative mitomycin C demonstrated $10 \%$ had late bleb leak, $10 \%$ scleral necrosis, and $5 \%$ hypotonous maculopathy. ${ }^{10}$ The incidence of endophthalmitis after antimetabolite augmented trabeculectomy is reported to be $1.1 \%$ and $3 \%$ for superiorly placed trabeculectomy and $8 \%$ and $9.4 \%$ for inferiorly placed trabeculectomies. ${ }^{12}{ }^{13}$ The long term risk of antimetabolite related endophthalmitis has yet to be fully evaluated, but it has been suggested that we may have a filtering surgery time bomb on our hands. ${ }^{14}$

However attractive a prospective plan to identify those more likely to fail, scarring is individual dependent and even cases thought to be at low risk may fail. Bleb encapsulation occurs in approximately $13 \%$ of eyes following trabeculectomy. ${ }^{15}$ Primary surgical manipulation under the surgical microscope has been shown to be effective in a series of nine of 13 cases $(69 \%) .{ }^{16}$ The addition of subconjunctival 5 -fluorouracil to the needling procedure increases success at relatively short term follow up. ${ }^{17}$ We are unaware of any literature regarding the number of patients who simply vascularise without encapsulation and fail.

This department has extensive experience with antiinflammatory fibrosis suppression in the modulation of wound healing after drainage by trabeculectomy and insertion of Molteno implants. Anti-inflammatory fibrosis suppression has three major advantages over local cytostatics. Antiinflammatory fibrosis suppression is only used when there are signs of bleb failure, the dose can be titrated according to the behaviour of the bleb, and it produces a diffusely spreading bleb with a minimal risk of late endophthalmitis or bleb rupture.

This series demonstrated a probability of 0.91 for IOP control with or without hypotensive medication at 8 years of follow up (see Fig 1). Other studies of the long term success of trabeculectomy in the management of primary open angle glaucoma provide almost identical results for similar periods of follow up. ${ }^{18-20}$ However, these studies are inclusive of all cases, both high and low risk, and not just high risk failing patients as in this series which demonstrated the same glaucoma success as the routine cases. The control of IOP, visual outcome, and incidence of subsequent cataract extraction in this series did not differ from that reported in a previous study of trabeculectomy outcomes at the same institution. ${ }^{20}$ In this small series of 63 patients nine reported minor gastric discomfort which was effectively treated by oral ranitidine without interrupting their anti-inflammatory fibrosis suppression medication. The response to anti-inflammatory fibrosis suppression was very consistent in the developing blebs over time and resulted in blebs of the relatively safe diffusely spreading type. So far, there has been no case of late hypotony, undue bleb thinning or infection.

\section{CONCLUSION}

When managing the postoperative course of filtering surgery and confronted with signs of bleb failure we recommend that anti-inflammatory fibrosis suppression be considered. We have found anti-inflammatory fibrosis suppression to be very effective in preventing fibrosis and bleb failure without significant local or systemic side effects.

\section{ACKNOWLEDGEMENT}

Financial support was obtained from the Healthcare Otago Charitable Trust. 


\section{Authors' affiliations}

J R Fuller, T H Bevin, A C B Molteno, Department of Ophthalmology, University of Otago Medical School, Dunedin, New Zealand

B J T Vote, Department of Ophthalmology, Dunedin Hospital, Dunedin, New Zealand

P Herbison, Department of Preventive and Social Medicine, University of Otago Medical School, Dunedin, New Zealand

Correspondence to: Professor A C B Molteno, Department of Ophthalmology, University of Otago Medical School, PO Box 913, Dunedin, New Zealand; georgi.bond@healthotago.co.nz

Accepted for publication 13 May 2002

\section{REFERENCES}

1 Skuta GL, Parrish RK 11 . Wound healing in glaucoma filtering surgery Surv Ophthalmol 1987;32:149-70.

2 Parrish RK II, Folberg R. Wound healing in glaucoma surgery. In: Ritch R, Shields MB, Krupin T, eds. The glaucomas. 2nd ed. Sydney: Mosby, 1996:1633-51.

3 Cordeiro MF, Gay JA, Khaw PT. Human anti-transforming growth factor- $\beta 2$ antibody: a new glaucoma anti-scarring agent. Invest Ophthalmol Vis Sci 1999:40:2225-34

4 Molteno ACB, Dempster AG. Methods of controlling bleb fibrosis around draining implants. Glaucoma. In: Mills KB, ed. Proceedings of the Fourth International Symposium of the Northern Eye Institute, Manchester, UK. Oxford: Pergamon Press, 1988:192-211.

5 Molteno ACB. New implant for glaucoma-clinical trial. Br Ophthalmol 1969;53:606-15.

6 Molteno ACB, Staughan JL, Ancker E. Control of bleb fibrosis after glaucoma surgery by anti-inflammatory agents. S Afr Med J 1976;50:881-5.

7 Molteno ACB. Mechanisms of intraocular inflammation. Trans Ophthalmol Soc N Z 1980;32:69-72.
8 The Fluorouracil Filtering Surgery Study Group. Five-year follow-up of the Fluorouracil Filtering Surgery Study. Am J Ophthalmol 1996; 121:349-66.

9 Rockwood EJ, Parrish RK II, Heuer DK, et al. Glaucoma filtering surgery with 5-fluorouracil. Ophthalmology 1987;94:1071-8.

10 Singh J, O'Brien C, Chawla HB. Success rate and complications of intraoperative $0.2 \mathrm{mg} / \mathrm{ml}$ mitomycin $\mathrm{C}$ in trabeculectomy surgery. Eye 1995;9:460-6

11 Bell RWD, Habib NE, O'Brien C. Long-term results and complications after trabeculectomy with a single per-operative application of 5-fluorouacil. Eye 1997;11:663-71.

12 Wolner B, Liebmann JM, Sassani JW, et al. Late bleb-related endophthalmitis afer trabeculectomy with adjunctive 5-fluorouracil. Ophthalmology 1991;98:1053-60.

13 Higginbotham EJ, Stevens RK, Musch DC, et al. Bleb-related endophthalmitis after trabeculectomy with mitomycin C. Ophthalmology 1996; 103:650-6.

14 Parrish R, Minckler D. "Late endophthalmitis" - filtering surgery time bomb? Ophthalmology 1996;103:1167-8.

15 Sherwood MB, Spaeth GL, Simmonds ST, et al. Cysts of Tenon's capsule following filtration surgery. Medical management. Arch Ophthalmol 1987; 105:1517-21.

16 Pederson JE, Smith SG. Surgical management of encapsulated filtering blebs. Ophthalmology 1985;92:955-8.

17 Allen LE, Manuchehri K, Corridan PG. The treatment of encapsulated trabeculectomy blebs in an out-patient setting using a needling technique and subconjunctival 5-fluorouracil injection. Eye 1998;12:119-23.

18 Ridgway AR. Trabeculectomy. A follow-up study. Br J Ophthalmol 1974;58:680-6.

19 Mills KB. Trabeculectomy: a retrospective long-term follow-up of 444 cases. Br J Ophthalmol 1981;65:790-5.

20 Molteno ACB, Bosma NJ, Kittleson JM, Otago Glaucoma Surgery Outcome Study-long-term results of trabeculectomy-1976 to 1995. Ophthalmology 1999;106:1742-50.

\section{Haploinsufficiency is the cause of dominant optic atrophy}

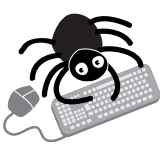

Please visit the British Journal of Ophthalmology website [www. bjophthalmol.com] for link to this full article. vidence from one family has, at last, pinpointed the cause of dominant optic atrophy (DOA) as an

— insufficiency in the OPA 1 gene. Previously, whether the disease arose through abnormal function-

- ing of altered proteins or insufficiency was just speculation.

One Australian family with DOA was studied in which no OPA 1 gene mutation had been identified. The ocular signs in the family members were typical of DOA of varying severity. The researchers genotyped the chromosomal DNA from as many of the family as possible with 12 microsatellite markers surrounding the OPA 1 gene and found that three flanking the gene were present as single alleles. After ruling out other causes it was clear the deletion of 560-860 kb DNA, resulting in the loss of one allele of OPA 1, was linked to DOA.

Fluorescence in situ hybridisation (FISH) was performed on metaphase chromosomes of three family members, with cloned OPA 1 c DNA as the probe. The results confirmed that one copy of the OPA 1 gene had been lost in the one affected member and one carrier, but not in the unaffected (control) member.

DOA is the commonest autosomal dominant inherited optic neuropathy. Most families with the disease have altered DNA in chromosome 3q28 (OPA 1), and more than 60 mutations have been identified at this locus. The researchers comment," haploinsufficiency of the OPA 1 protein is the cause of disease in this family and, in all likelihood, in other DOA families with truncated or mutated OPA 1 alleles."

\ J Med Genet 2002;39:e47 (http://www.jmedgenet.com/cgi/content/full/39/8/e47) 